\title{
Genetic Diversity and Relationships in Malus sp. Germplasm Collections as Determined by Randomly Amplified Polymorphic DNA
}

\author{
Nnadozie C. Oraguzie, ${ }^{1,2}$ Sue E. Gardiner, ${ }^{3}$ Heather C.M. Basset, ${ }^{3}$ Mirko Stefanati, ${ }^{1}$ \\ Rod D. Ball, ${ }^{4}$ Vincent G.M. Bus, ${ }^{1}$ and Allan G. White ${ }^{1}$ \\ Horticulture and Food Research Institute of New Zealand Limited, PB 1401 Goddard Lane, Havelock \\ North, New Zealand
}

\begin{abstract}
ADDiTIONAL INDEX wORDS. apple, RAPD markers, genetic diversity, analysis of molecular variance, cluster analysis, principal coordinate analysis

Abstract. Four subsets of apple (Malus Mill.) germplasm representing modern and old cultivars from the repository and apple genetics population of the Horticulture and Food Research Institute of New Zealand Limited were used in this study. A total of 155 genotypes randomly chosen from the four subsets were analyzed for random amplified polymorphic DNA (RAPD) variation. Nine decamer primers generated a total of 43 fragments, 42 of which were polymorphic across the 155 genotypes. Pairwise distances were calculated between germplasm subsets using the distance metric algorithm in S-PLUS, and used to examine intra-and inter-subset variance components by analysis of molecular variation (AMOVAR). A phenogram based on unweighted pair group method with arithmetic average (UPGMA) cluster analysis was constructed from the pairwise distances and a scatter plot was generated from principal coordinate analysis. The AMOVAR showed that most of the variation in the germplasm $(\mathbf{9 4 . 6 \%})$ was found within subsets, suggesting that there is significant variation among the germplasm. The grouping of genotypes based on the phenogram and scatter plot generally did not reflect the pedigree or provenance of the genotypes. It is possible that more RAPD markers are needed for determining genetic relationships in apple germplasm. Nevertheless, the variation observed in the study suggests that the current practice of sublining populations in the first generation to control inbreeding may not be necessary in subsequent generations. If these results are confirmed by fully informative molecular markers, germplasm managers should reassess the structure of their genetics populations. There may be a need to combine sublines in order to capture the maximum genetic diversity available and to streamline breeding efforts.
\end{abstract}

The consequences of loss of genetic diversity have been experienced sporadically throughout history, often with significant human and economic costs, which has been a common problem with many of the world's important food crops (Way et al., 1990). The genus Malus (apples), is characterized by large diversity, but thus far this has not been translated in the cultivated (Malus sylvestris (L) Mill. var. domestica (Borkh.) Mansf.) varieties (Way et al., 1990). Modern commercial apple orchards are dominated by only a few cultivars and many breeding programs utilize only a few well known cultivars in crosses for commercial apple production (Noiton and Shelbourne, 1992; Way et al., 1990). As a result, levels of inbreeding and coancestry in modern apples have been projected to increase (Noiton and Alspach, 1996).

Awareness of genetic diversity and management of crop genetic resources have been important components of plant improvement programs. The foundation of crop-based agriculture rests largely on the availability and knowledge of extant plant

Received for publication 10 July 2000. Accepted for publication 10 Jan. 2001. The research was partly funded by the New Zealand Foundation for Research, Science and Technology. We thank Madeleine Hofstee for her assistance with leaf collection and Erik Rikkerink, Lena Fraser and Trixie Harvey for critically reviewing the manuscript. The cost of publishing this paper was defrayed in part by the payment of page charges. Under postal regulations, this paper therefore must be hereby marked advertisement solely to indicate this fact.

${ }^{1}$ Horticulture and Food Research Institute of New Zealand Limited, PB 1401 Goddard Lane, Havelock North, New Zealand.

${ }^{2}$ Horticulture and Food Research Institute of New Zealand Limited, PB 1401 Goddard Lane, Havelock North, New Zealand. To whom all correspondence should be addressed; email: Noraguzie@hortresearch.co.nz.

${ }^{3}$ Horticulture and Food Research Institute of New Zealand Limited, Palmerston North Research Centre, Private Bag 11030, Palmerston North, New Zealand.

${ }^{4}$ Forest Research, Sala Street, Private Bag 3020, Rotorua, New Zealand. genetic resources in germplasm collections and successive stages of development in breeding programs. More specifically, knowledge of genetic diversity and relationships among sets of germplasm and the potential merit of the genetic diversity is critical to plant improvement (Lee, 1995).

In 1990, the Horticulture and Food Research Institute of New Zealand Limited (HortResearch), established a population improvement program to broaden the genetic base and, at the same time study the genetics of horticulturally important traits in apples. An apple genetics population was developed from imported seed and pollen from apple cultivars and varieties from clonal repositories in major apple producing regions in Europe, North America, Australia, Asia and South Africa (Noiton and Shelbourne, 1992). Seeds of accessions of Malus sieversii (Lebed.) were also obtained from Kazakhstan - the presumed center of origin of the cultivated apples (Hokanson et al., 1997). These seedlings have been planted in research orchards in a recurrent selection breeding strategy and maintained at multiple sites to allow for evaluation of general and regionally important horticultural traits and biotic and abiotic resistances. We as germplasm managers now need to assess the level of diversity within this collection to help make informed decisions on management procedures as well as on the choice of breeding strategies to use in combining horticulturally important traits in our breeding program.

Historically, assessments of genetic composition of germplasm collections have been carried out at several levels including inferences based on reproductive biology, ecogeographic data, morphology, pigmentation, ontogeny, social history, pedigree records, breeding behavior, in situ and ex situ evaluation of traits, chromosome structure and behavior, protein markers, and molecular markers such as restricted fragment length polymor- 
Table 1. Composition of provenances and the number of cultivars/selections assigned to each provenance based on species or geographic area of origin of the cultivar or maternal parent of the selection.

\begin{tabular}{|c|c|c|c|c|c|}
\hline \multirow[b]{2}{*}{ Provenance } & \multirow[b]{2}{*}{ Countries included } & \multicolumn{4}{|c|}{ Subset } \\
\hline & & 1 & 2 & 3 & 4 \\
\hline \multicolumn{6}{|l|}{ M. sylvestris var. domestica } \\
\hline North America & Canada, USA & 11 & 5 & 3 & 9 \\
\hline UK & England & 7 & 1 & 8 & 5 \\
\hline W. Europe & $\begin{array}{l}\text { Belgium, France, Denmark, Italy, Netherlands, Norway, Sweden, } \\
\text { Switzerland }\end{array}$ & 12 & 13 & 8 & 4 \\
\hline E. Europe & $\begin{array}{l}\text { Czechoslovakia, Estonia, Lithuania, Poland, Romania, } \\
\text { Former Soviet Union, Russia, Ukraine, Yugoslavia }\end{array}$ & 5 & 2 & 3 & 8 \\
\hline $\begin{array}{l}\text { S. Hemisphere/Japan } \\
\text { M. sieversii }\end{array}$ & New Zealand, Australia, Japan & 14 & 0 & 0 & 1 \\
\hline $\begin{array}{l}\text { Five accessions } \\
\text { M. kirghisorum }\end{array}$ & Dzhungarsky, Alatau, Karmenka Gorge Kazakhstan & 0 & 0 & 0 & 5 \\
\hline Three accessions & Ferganskiy Kherbet, Kyrgystan & 0 & 0 & 0 & 3 \\
\hline M. sieversii/M. kirghisorum & Dzhungarsky Alatau, Kazakhstan & 0 & 0 & 0 & 1 \\
\hline Species & $\begin{array}{l}\text { Malus sp. (other than } M \text {. sieversii) and crabapples irrespective of } \\
\text { country of origin }\end{array}$ & 0 & 6 & 2 & 6 \\
\hline Unknown & Provenance unknown or too uncertain & 1 & 3 & 6 & 3 \\
\hline & & 50 & 30 & 30 & 45 \\
\hline
\end{tabular}

phisms (RLFPs). The emergence of new polymerase chain reaction (PCR) based molecular markers, such as randomly amplified polymorphic DNA (RAPD) markers, simple sequence repeats (SSRs), and amplified fragment length polymorphisms (AFLPs), has created opportunities to characterize germplasm collections at the molecular level, with markers that are presumably selectively neutral and not affected in their expression by environmental effects. The increased levels of variation detectable with these molecular markers have allowed germplasm managers, plant breeders, and geneticists to pursue with new vigor important questions relating to crops and their genetic resources (Bretting and Widrlechner, 1995).

RAPD analyses have various scientific, operational, and budgetary advantages for genome mapping (Welsh and McClelland, 1990, 1991; Williams et al., 1990) and are used with a high degree of success as tools for harnessing genes from germplasm resources in our own apple genome mapping program (Gardiner et al., 1996a, 1999). These same advantages also appear valid for characterization of genetic variation for effective germplasm conservation and use, hence research in population genetics has shown the most growth with respect to the use of RAPD technology (Hedrick, 1992). RAPD markers have been used to create DNA fingerprints for the study of individual identity and taxonomic relationships in both eucaryotic and procaryotic organisms (Caetano-Anolles et al., 1991; Hu and Quiros, 1991; Kresovich et al., 1992); in apples, to differentiate cultivars (Koller et al., 1993), for paternity analysis (Harada et al., 1993), for determining genetic relationships in Malus sylvestris var. domestica cultivars and wild species (Dunemann et al., 1994), along with RFLPs and isozymes for tracing the parentage of 'Braeburn' (Gardiner et al., 1996b), and for phylogenetic studies (Zhou and Li, 2000).

The following research was conducted to examine the distribution of genetic diversity in the HortResearch apple germplasm collection using RAPD markers. The objectives of the investigation were to 1) assess the level of diversity between and within different subsets of apple germplasm, 2) determine the similarity of the selections/cultivars within the collections and 3) determine if the gene pool of apples had been effectively enlarged with the germplasm obtained from other countries and continents.

\section{Materials and Methods}

Plant material. The HortResearch apple germplasm was divided into four subsets. Subset 1 consisted of old cultivars and breeding lines that are maintained in the repository and the National Cultivar Center (NCC), and subsets 2, 3 and 4 consisted sublines in the apple genetics population. The apple genetics population comprised open-pollinated (op) families as well as families derived from controlled crosses from primitive cultivars, cider apples, and wild species (Noiton et al., 1999), including wild accessions of Malus sieversii (Lebed.) and M. kirghisorum (Al.) Ponom. from Kazakhstan. The families were divided into four sublines and planted at the third leaf stage in randomized incomplete blocks of 20 single tree plots at three different sites over a period of 4 years as follows: 1991: 224 families (10,232 trees); 1992: 149 families (12,800 trees); 72 families $(4,862$ trees); and 1994: 86 families (3,665 trees). Sublining was used to control inbreeding and maximize, as far as possible, genetic variability and heterozygosity within the population (Noiton and Shelbourne, 1992). We crossed within sublines per generation since inbreeding is unlikely to be a serious problem for many generations. Nevertheless, we had planned to introduce new unrelated material after a few generations to circumvent inbreeding. Ideally, the families should have been grouped into sublines based on region of origin so that individuals from the same region will be confined to one subline. For example, families from countries in Western Europe should be in a different subline than families from countries in North America or Eastern Europe. But this never really happened in our own case, due in part to the fact that seeds were not all received at the same time. Hence, the division into sublines was based on year of planting to the effect that a subline includes families from any region where seeds were obtained. However, assessing the diversity within the collection still has merit as the information will assist us in developing the best selection, breeding, and management strategy for the entire germplasm collection. We assigned subline 91 to subset 2, subline 92 to subset 3, and sublines 93 and 94 to subset 4 . At the time of this study, only families selected as parents for the second cycle of selections in the subline 91 were available. In total, 155 
Table 2. Names of families or cultivars from which selections were taken for this study, their field code, country of origin, and the year the cultivar was introduced according to the National Apple Register of the United Kingdom (Smith, 1971), U.S. Department of Agriculture Germplasm Resources Information Network (GRIN), and other sources.

\begin{tabular}{|c|c|c|c|}
\hline Name & Cultivar/family code & Origin & Year \\
\hline & & Families $^{\mathrm{z}}$ & \\
\hline Alexander (op) & 91.004 & Ukraine & 1600 \\
\hline Autumn Arctic (op) & 91.011 & USA & 1952 \\
\hline Belle de Lunteren (op) & 91.013 & Netherlands & \\
\hline Ben Davis (op) & 91.015 & USA & 1800 \\
\hline Bodil Neergård (op) & 91.020 & Denmark & 1850 \\
\hline Borsdorfer (op) & 91.022 & Germany & 1450 \\
\hline Calville d'Aout (op) & 91.026 & France & 1832 \\
\hline Cravert Rouge (op) & 91.037 & France & 1870 \\
\hline Druväpple (op) & 91.049 & Sweden & \\
\hline Grimes Golden (op) & 91.071 & USA & \\
\hline Gul Rickard (op) & 91.079 & Germany & \\
\hline Herfst Zoetzuur (op) & 91.083 & Unknown & \\
\hline Holovouske Malinove (op) & 91.085 & Unknown & \\
\hline John Downie (op) & 91.092 & UK & 1875 \\
\hline Linne's apple from Stenb (op) & 91.098 & Unknown & \\
\hline M. baccata 3 (ор). & 91.105 & Siberia & 1784 \\
\hline M. brevipes (op) & 91.107 & Japan & 1883 \\
\hline M. floribunda ( op) & 91.109 & Japan & 1862 \\
\hline M. sylvestris (op) & 91.111 & Europe & \\
\hline M. $\times$ atrosanguinea (op) & 91.112 & China & 1889 \\
\hline Robusta 5 ( op) & 91.113 & China & 1927 \\
\hline Reinette De Cusy (op) & 91.139 & France & 1863 \\
\hline Roman Stem (op) & 91.145 & USA & 1817 \\
\hline Rubin (op) & 91.150 & France & \\
\hline Szampanska (op) & 91.164 & Russia & 1852 \\
\hline White Angel (op) & 91.175 & USA & 1947 \\
\hline Zomerzuur (op) & 91.186 & Netherlands & \\
\hline Nonnetit Bastard x Bruyère & 91.212 & Denmark & 1700 \\
\hline Torstein $x$ De Casse & 91.220 & Norway & 1973 \\
\hline Transparente de Croncels x St Germain & 91.223 & France/Spain & $1869 / 1600$ \\
\hline Anurka (op) & 92.007 & Italy & \\
\hline Aromat de Vara (op) & 92.009 & Romania & \\
\hline Beachamwell (op) & 92.014 & UK & 1780 \\
\hline Bespodolenoe (op) & 92.019 & Unknown & \\
\hline Cluster Golden Pippin (op) & 92.026 & UK & 1750 \\
\hline Double Rose (Gros Api) (op) & 92.029 & France & 1628 \\
\hline Doux Normandie (op) & 92.030 & UK & 1783 \\
\hline Dutch Codlin (op) & 92.033 & Netherlands & \\
\hline Friandise (op) & 92.040 & Netherlands & 1760 \\
\hline Grahamovo (op) & 92.044 & England & \\
\hline Graue Herbstrenette (op) & 92.046 & England & \\
\hline Harvey (op) & 92.051 & UK & 1629 \\
\hline Hibernal (op) & 92.054 & USA & 1880 \\
\hline Håkonseple (op) & 92.058 & Germany & \\
\hline Kitaika Solotaja Rannaja (op) & 92.064 & Unknown & \\
\hline Leihmes (op) & 92.067 & Unknown & \\
\hline M. $\times$ hartwigii (op) & 92.075 & $U S A$ & 1906 \\
\hline M. ×robusta 'Persicifolia' (op) & 92.079 & China & 1910 \\
\hline Marie-Joseph d'Othee (op) & 92.081 & Belgium & 1947 \\
\hline Nezname Stave (op) & 92.088 & Unknown & \\
\hline Northwest Greening (op) & 92.090 & USA & 1872 \\
\hline Pozdni Kraje (op) & 92.105 & Unknown & \\
\hline Reinette Franche (op) & 92.110 & France & 1510 \\
\hline Rondestveit (op) & 92.113 & Unknown & \\
\hline
\end{tabular}


Table 2. Continued.

\begin{tabular}{|c|c|c|c|}
\hline Name & Cultivar/family code & Origin & Year \\
\hline Safranapfel (op) & 92.117 & Germany & $1544-1889$ \\
\hline Selena (op) & 92.121 & USA & 1864 \\
\hline Shafran Letnij (op) & 92.123 & Russia & \\
\hline Sussex Mother (op) & 92.133 & UK & 1800 \\
\hline Titovka (op) & 92.136 & Ukraine & 1870 \\
\hline Tower of Glamis (op) & 92.137 & UK & 1800 \\
\hline Bashkirian Beauty (op) & 93.003 & USSR & 1973 \\
\hline Binet Blanc Dore (op) & 93.006 & France & \\
\hline Blahova Ruzena (op) & 93.007 & Czechoslovakia & \\
\hline Dochdiany (op) & 93.018 & Poland & 1959 \\
\hline Gladstone (op) & 93.026 & UK & $1780-1883$ \\
\hline Golden Russet (op) & 93.028 & USA & 1800 \\
\hline Golden sweet (op) & 93.029 & USA & \\
\hline Joe Trio (op) & 93.034 & USA & 1936 \\
\hline Keo (op) & 93.035 & USA & 1940 \\
\hline Kola (op) & 93.037 & USA & \\
\hline Korichnoe Polosatoje (op) & 93.038 & Russia & $1831-1915$ \\
\hline Liestnaya Antonoffku 1836-A (op) & 93.041 & Unknown & \\
\hline Longfield (op) & 93.043 & Russia & 1870 \\
\hline Lord Derby (op) & 93.044 & UK & 1862 \\
\hline M. floribunda 821 (op) & 93.045 & Japan & 1908 \\
\hline M. honanensis (op) & 93.046 & China & 1913 \\
\hline M. prunifolia 19651 (op) & 93.047 & China & 1907 \\
\hline M. prunifolia 'Pendula' (op) & 93.048 & Species & \\
\hline Mammoth crab (op) & 93.049 & UK & 1939 \\
\hline Mildew Immune Seedling (op) & 93.051 & USA & \\
\hline Mleevskaya Crasavitsa (op) & 93.052 & Ukraine & \\
\hline Reinette Douce (op) & 93.059 & France & 1831 \\
\hline Rosemary Russet (op) & 93.060 & Unknown & \\
\hline Suisselpas Rozabols (op) & 93.062 & Unknown & \\
\hline Toellisaare (op) & 93.063 & Former Soviet Union & \\
\hline Trebu Seklandzis 7 (op) & 93.064 & Unknown & \\
\hline Uralian Winter Apple (op) & 93.065 & Russia & 1973 \\
\hline Vérité (op) & 93.066 & France & \\
\hline Worcester Pearmain $x$ Braeburn & 93.070 & UK/New Zealand & $1873 / 1956$ \\
\hline Yates (op) & 93.071 & USA & \\
\hline M. sieversii/kirghisorum GMAL 3539/3551y (op) & 94.016 & Kazakhstan & \\
\hline M. sieversii GMAL 3543 (op) & 94.020 & Kazakhstan & \\
\hline M. sieversii GMAL 3549 (op) & 94.026 & Kazakhstan & \\
\hline M. sieversii GMAL 3554 (op) & 94.031 & Kazakhstan & \\
\hline M. sieversii GMAL 3560 (op) & 94.034 & Kazakhstan & \\
\hline M. sieversii GMAL 3566 (op) & 94.038 & Kazakhstan & \\
\hline M. kirghisorum GMAL 3577 (op) & 94.046 & Kyrgystan & \\
\hline M. kirghisorum GMAL 3581 (op) & 94.050 & Kyrgystan & \\
\hline M. kirghisorum GMAL 3583 (op) & 94.052 & Kyrgystan & \\
\hline Braeburn (op) & 94.060 & New Zealand & 1956 \\
\hline Buchard (op) & 94.061 & Unknown & \\
\hline Commercio (op) & 94.064 & USA & 1865 \\
\hline Fogliona (op) & 94.067 & Italy & \\
\hline Joybells (op) & 94.077 & UK & 1908 \\
\hline Mother X Joybells & 94.080 & USA & 1914 \\
\hline
\end{tabular}

Table 2. continued next page.

genotypes were sampled randomly from the 4 subsets as follows: 50, 30, 30, and 45 genotypes respectively (Tables 1 and 2).

DNA ISOLATION. DNA was isolated using the method of Gardiner et al. (1996a, 1996b). The DNA was quantitated by visual comparison of DNA dilutions electrophoresed on $0.9 \%$ Ultrapure (Life Technologies, Gaithersburg, Md.) agarose gels in Trisacetate ethylenediaminetetraacetic acid (TAE) with $50 \mathrm{ng}$ of lambda phage DNA (Life Technologies) as standard. Electrophoresis runs were for $1.5 \mathrm{~h}$ at $2.5 \mathrm{~V} \cdot \mathrm{cm}^{-1}$, and DNA staining was with 0.35 $\mu \mathrm{g} \cdot \mathrm{mL}^{-1}$ ethidium bromide. Under these conditions, apple DNA runs as a clear band at the same position on the gel as the standard.

DNA AMPLIFICATION. Four hundred decamer oligonucletide primers (Operon Technologies, Alameda, Calif.) including the 39 already developed for 'Braeburn' by Gardiner et al. (1996b), were 
Table 2. Continued.

\begin{tabular}{|c|c|c|c|}
\hline Name & Cultivar/family code & Origin & Year \\
\hline & & Cultivars & \\
\hline Cox's Orange Pippin & $\operatorname{NCC~} 1^{x}$ & UK & 1850 \\
\hline Pacific Beauty & NCC 2 & New Zealand & 1995 \\
\hline Pacific Queen & NCC 3 & New Zealand & 1995 \\
\hline Pacific Rose & NCC 4 & New Zealand & 1995 \\
\hline Pink Lady & NCC 5 & Australia & \\
\hline Southern Snap & NCC 6 & New Zealand & 1995 \\
\hline McIntosh & R13T229 & Canada & 1796 \\
\hline Delicious & R18T357-367 & USA & 1880 \\
\hline Red Chief & R1T10 & USA & 1972 \\
\hline Hi Early Red & R1T22 & USA & 1955 \\
\hline Fuji1-1 & R1T34 & Japan & 1962 \\
\hline Antonovka & R1T46 & Russia & $<1862$ \\
\hline Braeburn & R1T54 & New Zealand & 1956 \\
\hline Granny Smith & R1T56 & Australia & 1868 \\
\hline Royal Gala & R22T558-614 & New Zealand & 1965 \\
\hline Kentish Fillbasket & R2T13 & UK & 1820 \\
\hline Golden Delicious & R2T29 & USA & 1890 \\
\hline Ballarat Seedling & R2T47 & Australia & 1900 \\
\hline Close & R2T47 & USA & 1925 \\
\hline Democrat & R2T49 & Australia & 1900 \\
\hline Reinette du Canada & R2T56 & France & 1771 \\
\hline Kapai Red Jonathan & R30T966 & USA & 1826 \\
\hline Nonpareil & R3T15 & France & 1500 \\
\hline Fraasov Letni & R3T27 & Czechoslovakia & \\
\hline Nonnetit Bastard & R3T3 & Denmark & 1700 \\
\hline Alkmene & R3T39 & Germany & 1965 \\
\hline Shafran Kitajka & R3T51 & Russia & 1907 \\
\hline Alma Pippin & R4T20 & South Africa & \\
\hline Adam's Pearmain & R4T32 & UK & 1826 \\
\hline Rae Ime & R4T44 & Estonia & \\
\hline Flueritard Rouge & R4T56 & France & 1950 \\
\hline Rymers & R4T7 & UK & 1818 \\
\hline Cortland & R5T13 & USA & 1951 \\
\hline Rheinischer Krummstiel & R5T25 & Germany & 1821 \\
\hline Geheimrat Breuhahn & R5T37 & Germany & 1895 \\
\hline Maggi Grieve & R5T49 & UK & 1928 \\
\hline Idared & R5T59-60 & USA & \\
\hline Mr Fitch & R6T1 & New Zealand & \\
\hline Red Melba & R6T13 & Canada & \\
\hline Hladikovo Preuradne & R6T25 & Czechoslovakia & \\
\hline Guldborg & R6T37 & Denmark & 1870 \\
\hline Tydeman's Early Worcester & R6T49 & UK & 1929 \\
\hline Jamba & R7T14 & Germany & 1954 \\
\hline Mettias & R7T2 & Unknown & \\
\hline Lady Williams & R7T26 & Australia & 1935 \\
\hline Peasgood Nonsuch & R7T44 & UK & 1858 \\
\hline Pfirsichroter Sommerapfel & R7T56 & Germany & 1800 \\
\hline Reintte Marbree d'Auvergne & R8T33 & France & \\
\hline Redwell & R8T45 & USA & 1911 \\
\hline Glockenapfel Eckter & R8T9 & Germany & 1948 \\
\hline
\end{tabular}

${ }^{\mathrm{z}} \mathrm{Op}=$ open pollinated.

${ }^{\mathrm{y}}$ GMAL = Geneva Malus; A GMAL number references a local number assigned by the USDA, ARS Plant Genetic Resources Unit, Cornell University Geneva, N.Y., to an accession that has no plant introduction number.

${ }^{\mathrm{x}} \mathrm{NCC}=$ National Cultivar Center, and RT $=$ row/tree number for cultivars in the repository.

prescreened using five cultivars ('Cox's Orange Pippin', 'Southern Snap', 'Alkmene', 'Fogliona' and 'Braeburn'), to select primers that amplified reliably. Out of these, nine that produced polymorphic, reproducible, and scorable bands were chosen for further amplification of DNA from all genotypes (Table 3).

The RAPD-PCR runs generally were performed once; if there 
Table 3. Summary of RAPD bands scored.

\begin{tabular}{lccc}
\hline \hline & & $\begin{array}{c}\text { Bands } \\
\text { produced/ } \\
\text { primer } \\
\text { (no.) }\end{array}$ & $\begin{array}{c}\text { Polymorphic } \\
\text { bands produced/ } \\
\text { primer } \\
\text { (no.) }\end{array}$ \\
\hline OPC06 & GAACGuence & 6 & 6 \\
OPC10 & TGTCTGGGTG & 4 & 4 \\
OPC13 & AAGCCTCGTC & 5 & 5 \\
OPD03 & GTCGCCGTCA & 5 & 5 \\
OPD20 & ACCCGGTCAC & 4 & 3 \\
OPR16 & CTCTGCGCGT & 4 & 4 \\
OPK14 & CCCGCTACAC & 5 & 5 \\
OPU08 & GGCGAAGGTT & 6 & 6 \\
OPQ09 & GGCTAACCGA & 4 & 4 \\
\hline
\end{tabular}

was a reaction failure for a particular primer in one or more lanes, the run was repeated. The reaction mixtures $(16.5 \mathrm{uL})$ contained $10 \mathrm{~mm}$ tris-HCL (pH 8.8), $50 \mathrm{~mm} \mathrm{KCl}, 2.0 \mathrm{~mm} \mathrm{MgCl}_{2}, 0.01 \%$ gelatin, $0.8 \%$ formamide, $0.1 \mathrm{~mm}$ each dNTP, $0.2 \mathrm{~mm}$ each primer and $1.5 \mathrm{ng}$ of apple genomic DNA, 1.2units of Taq DNA polymerase (Stratagene, La Jolla, Calif.) and were overlaid with $8 \mu \mathrm{L}$ paraffin. Reactions were set up in 96 well plates using a Biomek 2000 Laboratory Automation Workstation (Beckmann, Fullerton, Calif.) that also loaded reaction products on electrophoresis gels. Amplification was conducted in a thermal cycler (PCR Express; Hybaid, Ashford, United Kingdom) programmed for $4.5 \mathrm{~min}$ at $94^{\circ} \mathrm{C}, 40$ cycles of $1 \mathrm{~min}$ at $94{ }^{\circ} \mathrm{C}, 1 \mathrm{~min}$ at $37^{\circ} \mathrm{C}$, and $2 \mathrm{~min}$ at $72^{\circ} \mathrm{C}$, followed by a final extension cycle of $10 \mathrm{~min}$ at $72{ }^{\circ} \mathrm{C}$. These reaction conditions produce consistently reproducible results for a range of apple DNA concentrations in our laboratory. Amplification products were electrophoresed on gels consisting of a mixture of $0.45 \%$ Separide (Life Technologies), $40.22 \%$ UltraPure agarose (Life Technologies) and $0.22 \%$ Nusieve GTG agarose (FMC, Rockland, Maine) in TAE at $4 \mathrm{~V} \cdot \mathrm{cm}^{-1}$ for $\approx 5$ h. Gels were stained with ethidium bromide at $0.35 \mu \mathrm{g} \cdot \mathrm{mL}^{-1}$, visualized under ultra violet light and photographed in color (64T Kodak Ektachrome film; Eastman Kodak, Rochester, N.Y.). RAPD product sizes were estimated using a 100-base pair (bp) DNA standard ladder (Life Technologies). Reproducibility between gels and consistency of scoring was checked by effectively overlapping the gels-five samples, including 'Cox's Orange Pippin', 'Southern Snap', 'Alkmene', 'Fogliona' and 'Braeburn', were repeated on each 42 well gel.

DATA ANALYSIS. Individual RAPD bands were scored as present or absent from enlarged color photographs. Faint bands of doubtful reproducibility were ignored, and those that were very faint compared to others in the same position were scored as absent $(0)$ while the bright ones were scored as present (1).

All analyses were performed in S-Plus (Statistical Sciences, Inc., 1995) using the methods of Becker et al. (1988) and Chambers and Hastie (1992). Genetic distance was calculated using the 'binary' metric option. The distance (D) between any 2 genotypes is given by: $D=$ (number of bands in common)/(total number of bands). In this way, no assumptions were made on the number of loci or alleles. The distances were used to construct a phenogram using the unweighted pair group average (UPGMA) hierarchical clustering method.

Principal coordinate analysis was done with the presence and absence data matrix to obtain a graphical representation of the genotypes in dimension and space. It was done such that the Euclidean distance between genotypes was about the same as the
Table 4. Frequency of polymorphic RAPD bands in four subsets of New Zealand apple germplasm. $\mathrm{P}$ is the proportion of polymorphic bands for each primer. $P t$ is the overall proportion of polymorphic bands in each subset.

\begin{tabular}{|c|c|c|c|c|}
\hline \multirow[b]{2}{*}{ Primer band } & \multicolumn{4}{|c|}{$\mathrm{P}$} \\
\hline & Subset 1 & Subset 2 & Subset 3 & Subset 4 \\
\hline$\overline{\mathrm{OPC} 06 \_1}$ & 42 & 23 & 27 & 36 \\
\hline OPC06_2 & 46 & 27 & 53 & 29 \\
\hline OPC06_3 & 62 & 57 & 33 & 67 \\
\hline OPC06_4 & 36 & 47 & 57 & 63 \\
\hline OPC06_5 & 60 & 40 & 20 & 40 \\
\hline OPC06_6 & 60 & 23 & 47 & 63 \\
\hline$P t$ & 100 & 100 & 100 & 100 \\
\hline OPC10_1 & 10 & 27 & 27 & 24 \\
\hline OPC10_2 & 88 & 90 & 90 & 89 \\
\hline OPC10_3 & 46 & 50 & 43 & 42 \\
\hline OPC10_4 & 38 & 37 & 30 & 47 \\
\hline$P t$ & 100 & 100 & 100 & 100 \\
\hline OPC13_1 & 16 & 10 & 0 & 13 \\
\hline OPC13_2 & 50 & 67 & 47 & 42 \\
\hline OPC13_3 & 24 & 23 & 20 & 11 \\
\hline OPC13_4 & 8 & 0 & 0 & 0 \\
\hline OPC13_5 & 52 & 3 & 40 & 44 \\
\hline$P t$ & 100 & 100 & 100 & 100 \\
\hline OPD03_1 & 54 & 63 & 30 & 40 \\
\hline OPD03_2 & 24 & 57 & 23 & 29 \\
\hline OPD03_3 & 12 & 0 & 7 & 18 \\
\hline OPD03_4 & 100 & 93 & 97 & 91 \\
\hline OPD03_5 & 24 & 23 & 37 & 22 \\
\hline$P t$ & 100 & 60 & 100 & 100 \\
\hline OPD20_1 & 16 & 13 & 20 & 9 \\
\hline OPD20_2 & 12 & 37 & 17 & 9 \\
\hline OPD20_3 & 100 & 100 & 100 & 100 \\
\hline OPD20_4 & 44 & 27 & 30 & 44 \\
\hline$P t$ & 75 & 75 & 75 & 75 \\
\hline OPK14_1 & 14 & 7 & 13 & 16 \\
\hline OPK14_2 & 2 & 0 & 3 & 7 \\
\hline OPK14_3 & 30 & 10 & 33 & 64 \\
\hline OPK14_4 & 32 & 7 & 17 & 43 \\
\hline OPK14_5 & 44 & 30 & 30 & 90 \\
\hline$P t$ & 100 & 90 & 100 & 100 \\
\hline OPQ09_1 & 52 & 50 & 77 & 58 \\
\hline OPQ09_2 & 90 & 100 & 80 & 89 \\
\hline OPQ09_3 & 78 & 70 & 83 & 69 \\
\hline OPQ09_4 & 20 & 17 & 27 & 20 \\
\hline$P t$ & 100 & 100 & 100 & 100 \\
\hline OPR16_1 & 26 & 33 & 47 & 24 \\
\hline OPR16_2 & 32 & 63 & 70 & 51 \\
\hline OPR16_3 & 4 & 3 & 30 & 13 \\
\hline OPR16_4 & 0 & 13 & 7 & 20 \\
\hline$P t$ & 100 & 100 & 100 & 100 \\
\hline OPU08_1 & 70 & 50 & 77 & 53 \\
\hline OPU08_2 & 42 & 73 & 37 & 71 \\
\hline OPU08_3 & 62 & 30 & 70 & 73 \\
\hline OPU08_4 & 44 & 27 & 20 & 42 \\
\hline OPU08_5 & 32 & 33 & 20 & 38 \\
\hline OPU08_6 & 16 & 7 & 17 & 16 \\
\hline$P t$ & 100 & 100 & 100 & 100 \\
\hline
\end{tabular}




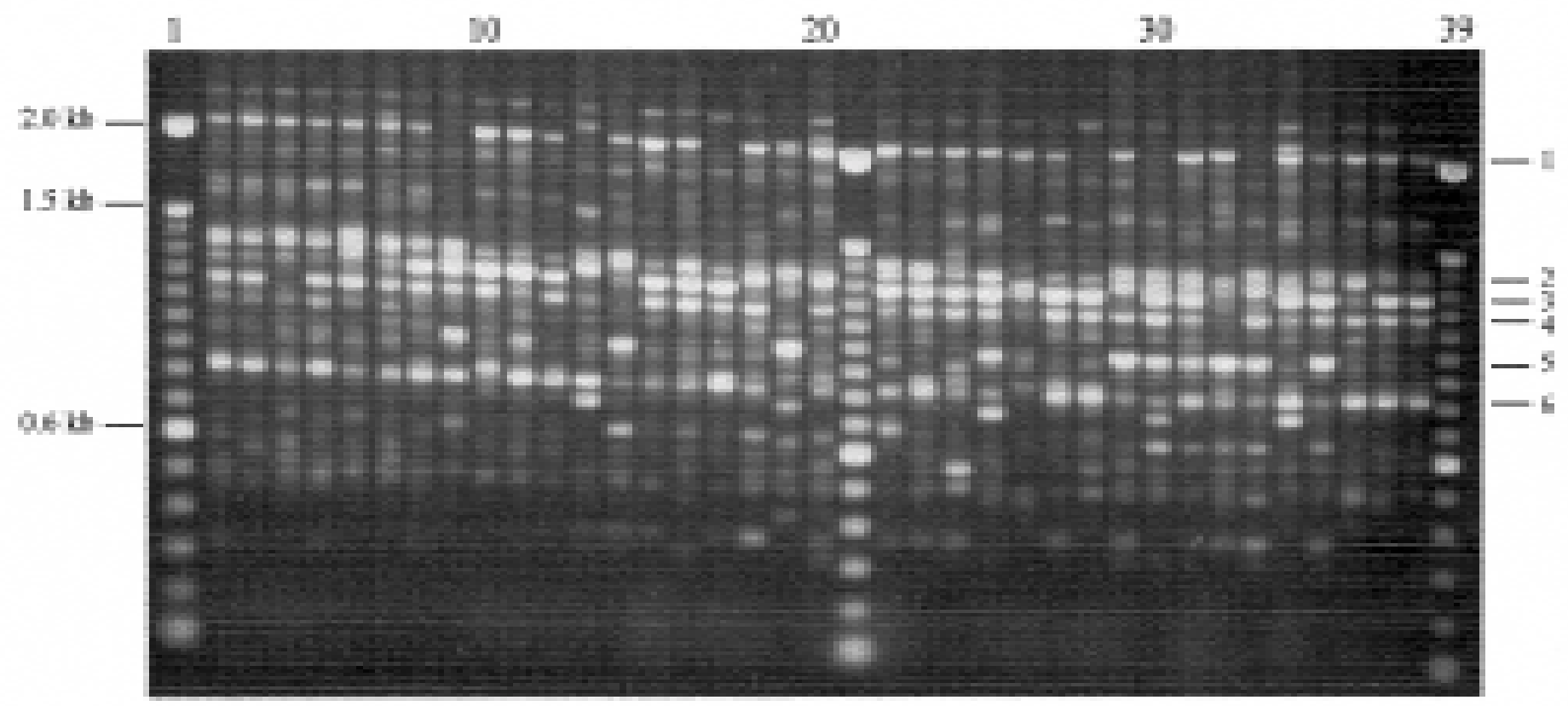

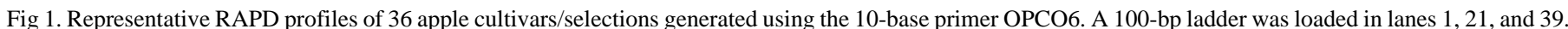

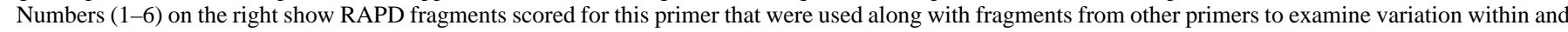

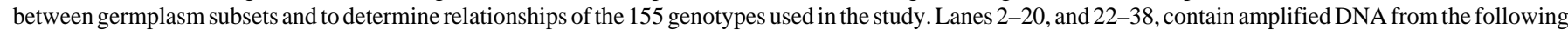

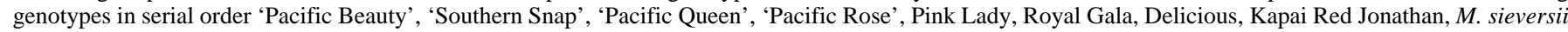

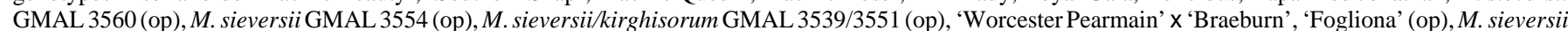

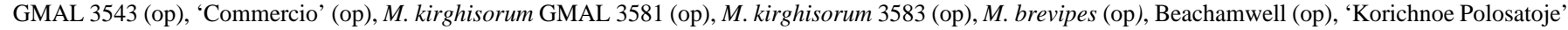

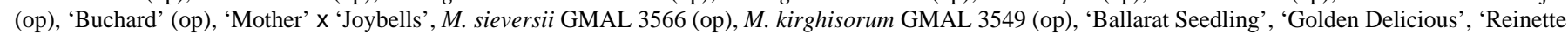
du Canada', 'Kentish Fill basket', 'Granny Smith', 'Braeburn', 'Antonovka', 'Fuji-1', 'Hi Early Red' and 'Red Chief'.

raw pairwise distances between genotypes.

A V-fold cross validation with $\mathrm{V}=10$, was used to choose the number of variables to use for analysis of variance (ANOVA). This involved choosing a random sample of $\mathrm{V}$ points to be left out of the model for the fitting process (calculating distance matrix and multidimensional scaling). For various values of the number of principal coordinates, $\mathrm{k}$, the cross validation stress defined as the mean squared error or relative errors in pairwise distances, mean [(d.obs-d.pred)/d.pred)^2)], was calculated. Where, d.obs, denotes the observed distance and d.pred, the predicted distance, for a given value of $k$. The process was repeated 15 times giving 15 stress values. The cross validation stress values continued to decrease almost until $k=30$. Values for $k>24$ could not be distinguished statistically.

Analysis of molecular variance (AMOVAR) was carried out with 24 principal coordinates as well as with all 43 , using the AMOVAR procedure in S-PLUS to obtain gene diversity indices for all individuals within subsets and among subsets. The variance and the sums of squares of these analyses were combined to give a pooled analysis. Variance components were calculated from the mean squares using the method of moments estimates.

The $\mathrm{F}$ statistic $\left(F_{s t}\right)$ which is a measure of reduction in the number of heterozygotes, was computed using the equation $F_{s t}=$ $\sigma_{\mathrm{p}}^{2} /\left(\sigma_{\mathrm{p}}^{2}+\sigma_{\mathrm{G}}^{2}\right)$ (Excoffier et al., 1992).

\section{Results}

From the nine random primers used (Table 3), 43 amplification products (bands) were stably generated (Table 4 ), 42 of which were polymorphic, thus reflecting the genetic diversity within the germplasm. Primer OPD20 produced a fragment (OPD20_3) of size between 1 and $1.1 \mathrm{~kb}$, which was common to genotypes in all subsets. One band from primer OPC13, OPC13_4, was unique to subset 1 and most (89\%) of the other bands were present in all subsets but in varying frequencies. One band each was fixed in subsets 1 and 2, the band OPD03_4 in subset 1 and OPQ09_2, in subset 2 . The level of intrasubset polymorphism varied from one RAPD primer to the other and from one subset to the other.

The polymorphisms observed with 36 representative genotypes using primer OPCO6 is illustrated in Fig. 1. Though most of the bands were found in the majority of genotypes, no single fragment was common to all 36 genotypes. 'Pacific Beauty' and 'Southern Snap' had identical profiles (lanes 2 and 3) while 'Pacific Queen' (lane 4) lacked the fragment, OPC06_4, which was common to the rest of 'Gala' $x$ 'Splendour' cultivars. 'Pacific Rose' shared a fragment of size 1.5 to $2.0 \mathrm{~kb}$ with 'Gala' (one of the parents), which was lacking in the other GS cultivars. The putative two sports of 'Delicious', 'Red Chief' and 'Hi early Red' (lanes 37 and 38) differed by one band (OPC06_1) and both lacked fragment OPC06_2 observed in 'Delicious' (lane 8).

The genetic distance between genotypes was generally high (data not presented), with most distance measures falling between 0.52 and 0.73 . The highest was 0.952 and the lowest 0.148 . It was not uncommon for individuals from different subsets to have a high genetic similarity. For example, 'Ballarat Seedling' (subset 1 ) and $M \times$ atrosanguinea (op) in subset 2 had a similarity of $78.9 \%$ compared to a similarity of $4.8 \%$ between 'Anurka' (op). and M. Xrobusta 'Persicifolia' Rehder (op). both from subset 3 .

The phenogram (Fig. 2) from UPGMA cluster analysis showed several groupings which did not agree with the provenance or ancestry of the genotypes. However, it is difficult to establish the precise pedigree of the majority of apple cultivars, and even particularly the open-pollinated families used in subsets 2 to 4 . 


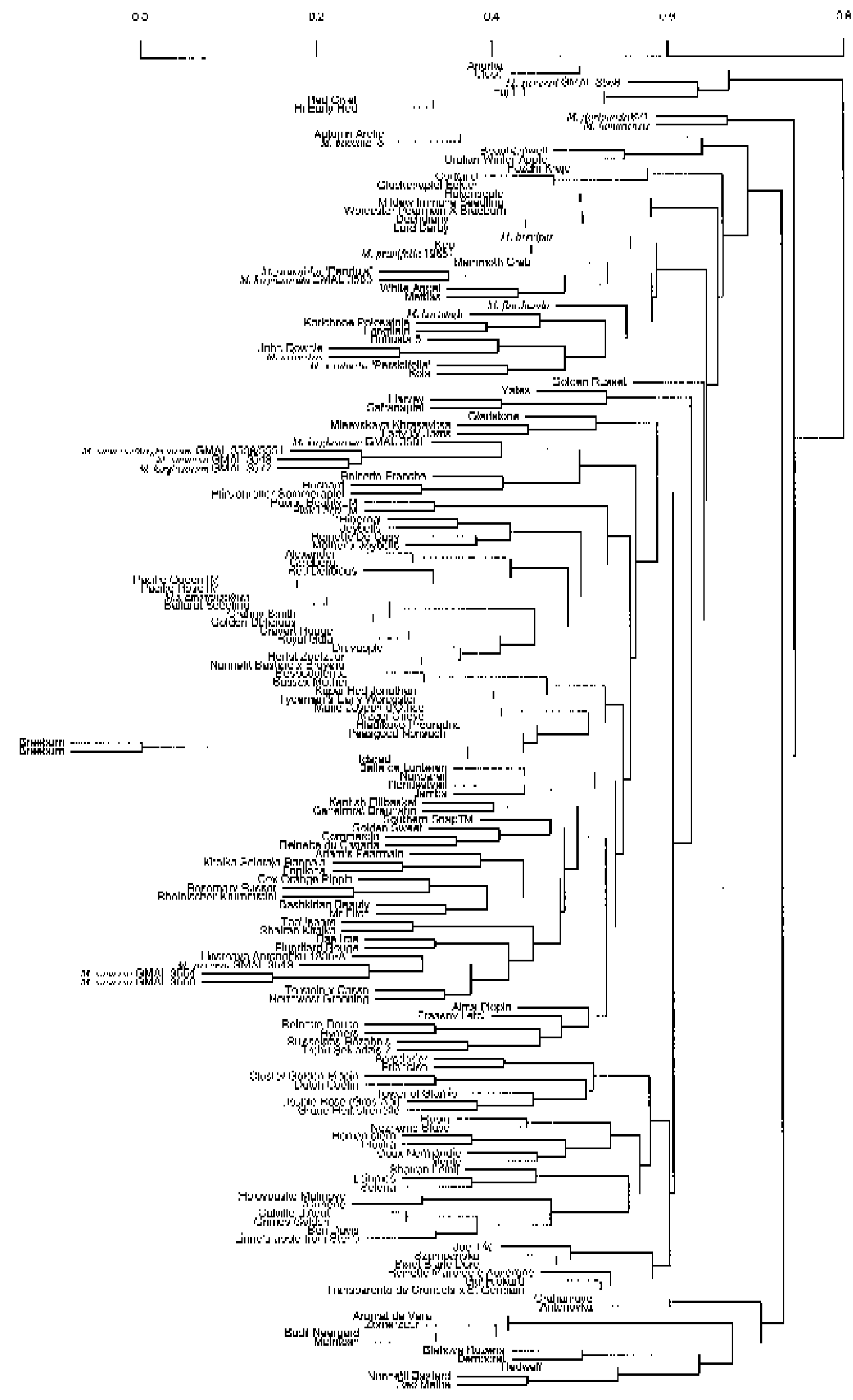

Fig. 2. Phenogram from UPGMA cluster analysis of 155 apple genotypes based on their phenotypes for 43 RAPD bands. 


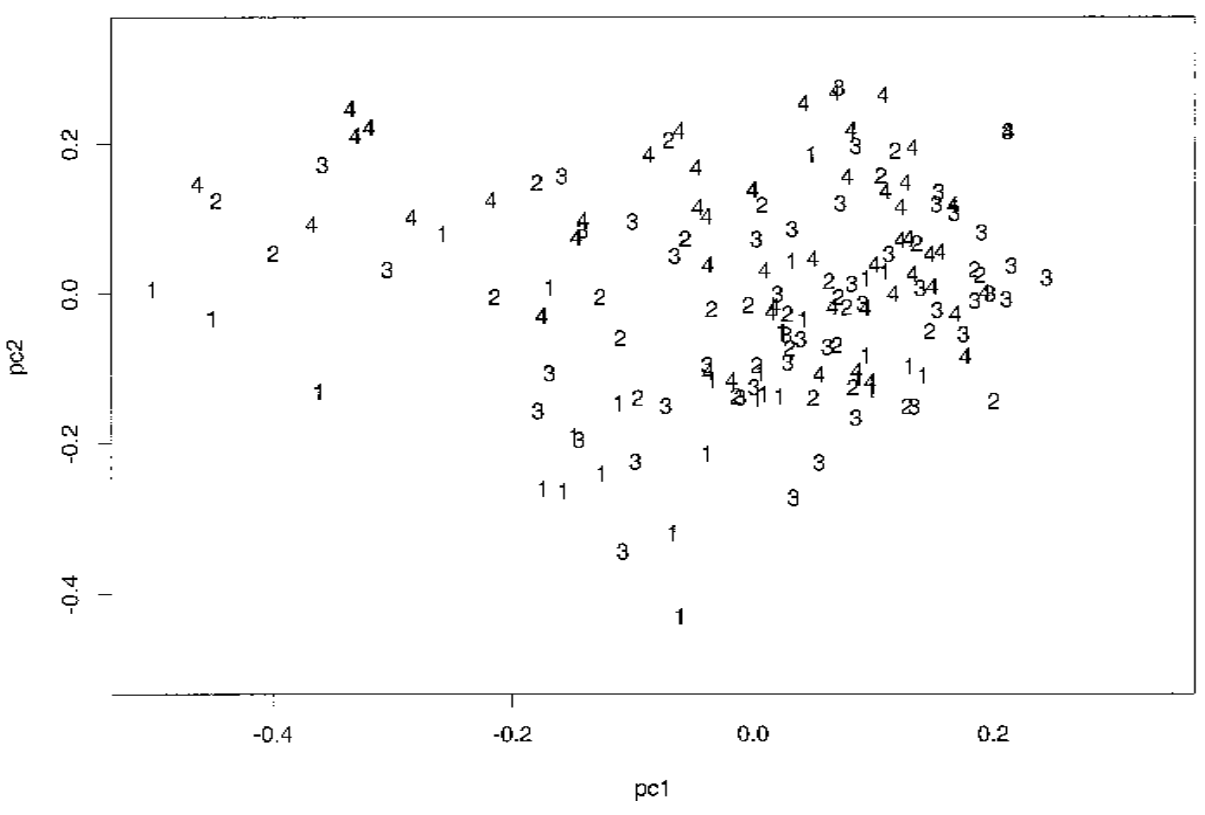

Fig. 3. Scatter plot of the first two principal coordinates of the 43 RAPD bands matrix.

The GS cultivars however, had 3 ('Pacific Beauty', 'Pacific Queen' and 'Pacific Rose') out of the four used for the study grouped together with 'Royal Gala'. 'Southern Snap' was placed in another group starting with 'Belle de Lunteren' through to 'Northwest Greening'. This placement of one of the sibs is not inconsistent with the relationship of 'Cox's Orange Pippin' to 'Gala' ['Gala' = 'Kidd's Orange' ('Delicious' x 'Cox's Orange Pippin') $x$ 'Golden Delicious']. 'Braeburn' is recognized as having a Cox-type flavor and cultivars known or suspected to have 'Cox' in their parentage occurred in the grouping starting from 'Pacific Beauty' to 'Trebu Sekladzis 7' ('Pacific Beauty', 'Delicious', 'Pacific Queen', 'Pacific Rose', 'Royal Gala' and 'Cox' itself). Duplicate 'Braeburn' samples were not separable. The group starting with M. floribunda 821 through to 'Kola' contains many selections and families frequently used as parents in our resistance breeding program. The majority of genotypes in this group are crabapples.

Out of a total of 43 bands representing 43 principal coordinates, 16 principal coordinates explained $90 \%$ of the variation between subsets while 33 explained $99 \%$ of the variation. A plot of the first two principal coordinates (Fig. 3) which accounted for $38 \%$ of the variation did not separate out the subsets into distinct groups based on provenance or country of origin. The grouping of the individuals was similar to that obtained with the phenogram. Genotypes, irrespective of the subset they belonged to, were scattered all over the plot. However, in the lower left half of the plot, only a few genotypes were found and these were mostly from subsets 1 and 3. A plot of the first six principal coordinates, which explained 64\% of the variation in pairs (plot not presented) did not improve the groupings.

The AMOVAR results obtained with 24 and 43 RAPD markers were very close, so the results presented herein are for the 43 RAPD markers (Table 5). Although the $\mathrm{F}$ test for difference shows weak evidence for a variation among subsets (a $P$ value of 0.0381 ), examination of the between subset variance component in Table 5 shows that this difference is not major. Most of the total genetic diversity (94.6\%) was found within subsets. This pooled within subset variation was distributed almost equally among the subsets irrespective of the composition and differing number of individuals in the subsets.

\section{Discussion}

Results indicate there are high levels of genetic variation within the HortResearch apple germplasm. Partitioning this variation into its within- and between-subset components indicated that $94.6 \%$ was maintained within subsets. This higher within subset diversity agrees with the finding of Lamboy et al. (1996) that $M$. sieversii accessions maintained $85 \%$ of their diversity within regions compared to $15 \%$ between regions. This study was not designed to specifically investigate population structure, and hence may not be directly comparable to such studies. Nevertheless, the results agree with the general observation that woody perennial outbreeding species maintain most of their variation within populations (Hamrick, 1994). Hamrick (1994) outlined a number of life history traits that affect partitioning of genetic diversity in tree species. These can be summarized in four general observations. First, temperate species on average, display higher levels of variation than tropical trees. Second, species with a widespread geographical distribution tend to display greater diversity than those of a more limited range. Also, taxa that are predominantly outcrossing maintain higher levels of genetic diversity than those that are primarily autogamous. Finally, selfing species tend to show a high degree of population substructuring, with diversity maintained among rather than within populations. Apparently, these four observations are true for apples and could well explain the high levels of variation obtained in this study.

The between subset component accounted for only $\approx 5 \%(P=$

Table 5. Analysis of molecular variance (AMOVAR) based on 43 RAPD bands for 155 selections/cultivars of apples grouped into four subsets. The estimated within germplasm subset variation (\%) is 0.244 for Subset 1, 0.245 for Subset 2, 0.242 for Subset 3, and 0.242 for Subset 4 .

\begin{tabular}{|c|c|c|c|c|c|c|c|c|}
\hline $\begin{array}{l}\text { Source of } \\
\text { variation }\end{array}$ & $\mathrm{dF}$ & SS & MS & $\begin{array}{c}\text { Variation } \\
(\%)\end{array}$ & $\mathrm{F}^{\mathrm{Z}}$ & 1-Pr(F) & Vcomp $^{y}$ & $F_{s t}{ }^{\mathrm{x}}$ \\
\hline Between subset & 3 & 2.1 & 0.699 & 0.054 & 2.88 & 0.0381 & 0.012 & 0.066 \\
\hline Within subset & 151 & 36.7 & 0.243 & 0.946 & $\mathrm{NA}^{\mathrm{w}}$ & NA & 0.243 & NA \\
\hline
\end{tabular}

${ }^{\mathrm{z}}$ Variance ratio.

yVariance component.

${ }^{\mathrm{x}} \mathrm{F}$ statistic.

${ }^{\mathrm{w}} \mathrm{NA}=$ not applicable. 
0.038) of the genetic variation between genotypes. This apparent lack of differentiation between the subsets could be attributed to high levels of mating and genetic recombination. Further evidence for lack of differentiation between subsets comes from the fact that all the subsets have most of the bands in common (though in different frequencies) and only one subset (subset 1, old cultivars and breeding lines) has a private allele (band, OPC13_4). One other possible explanation for the similarities between the subsets may be because of comigration of nonhomologous RAPD bands (Dunemann et al., 1994; Rieseberg, 1996). Though, Kazan et al. (1993) suggested that, within a genus, it is likely that fragments shared by any two species will be allelic. So, this theory needs further investigation. The similarities may also be caused by paralogous evolution or the wide range of variation across the species range. In any case, the open-pollinated nature of most of the material used would tend to enhance the similarities.

It was interesting to find that there was the same amount of genetic variation within subsets irrespective of the size and composition of each subset. We expected subset 1 to have much less variation than other subsets due to it containing only $M$. $\times$ domestica genotypes including the five cultivars ('Cox's Orange Pippin', 'McIntosh', 'Delicious', 'Golden Delicious' and 'Jonathan') reported by Noiton and Alspach (1996) as contributing to the pedigree of modern apple cultivars. Also, all the genotypes were cultivars that have been clonally propagated. Other subsets had varying numbers of $M$. sylvestris var. domestica and other Malus species (largely open pollinated). Apparently, the high levels of coancestry reported in modern apples (Noiton and Alspach, 1996; Way et al., 1990) are not obvious in this subset. Dunemann et al. (1994) observed a high degree of variation between $M$. ×sylvestris var domestica accessions with RAPD markers and suggested that a broad genetic base still exists in cultivated apples. Hokanson et al. (1998) using 8 microsatellite (SSR) markers detected high levels of variation with a mean of 12.1 alleles per locus with 66 accessions of $M$. sylvestris var. domestica from the U.S. Department of Agriculture germplasm collection. This germplasm included the 5 cultivars that have been used as recurrent parents in modern commercial apple breeding (Noiton and Alspach, 1996), as well as some old cultivars. We have reviewed our fruit evaluation database and found that the $M$. sieversii genotypes (op) used in this study (in subset 4) are all edible and have fruit about the same size as the cultivated varieties. Hence, as far as fruit size is concerned, subset 4 may not be much different from subset 1 . This observation is in line with that of Juniper et al. (1999) where the fruit of $M$. sieversii show an extensive diversity from almost inedible crabs to fruit not very dissimilar to some modern cultivars. These results suggest there is still an appreciable amount of diversity within $M$. sylvestris var. domestica, which perhaps is retained in the old cultivars. It should be noted here that many apple cultivars are open pollinated seedlings and are of unknown or, at least, uncertain origin. Therefore, even among some widely grown cultivars, the genetic relationships are not yet fully known. It can be assumed that $M$. sylvestris var. domestica is a complex hybrid with several, as yet not fully identified, species involved in the process of domestication (Korban and Skirvin, 1984; Way et al., 1990). Hence, the genetic base of cultivated apples is probably still very broad.

The phenogram from UPGMA cluster analysis using the 155 genotypes produced several groupings most of which could not be explained by the pedigree or provenance of the genotypes. The estimated similarity between the genotypes was from 0.048 [between 'Anurka' (op). and M. ×robusta 'Persicifolia' (op)] to
0.85 (between 'Cox Orange Pippin' and 'Pacific Beauty') (data not presented). This range of similarities is very wide, therefore, we believe that these similarities are the result of the high polymorphism revealed by the RAPD markers. Other causes such as the approach taken to analyze the data, which involved scoring each band as a unique character and estimating the distances (or dissimilarities) using the shared band method, may not be ruled out. Although, the shared band method may not be a problem with identical clones since the two 'Braeburns', the cultivar in subset 1 and the seedling derived from this cultivar, in subset 4 , had a genetic similarity of $100 \%$. Nevertheless, the use of shared band analysis to investigate phenetic relationships in 18 potato (Solanum tuberosum L.) cultivars by Provan et al. (1996), produced groupings among the cultivars which did not agree with their coancestry, though the authors found sufficient polymorphism with the 16 SSR primers used which clearly distinguished between the cultivars. Dunemann et al. (1994) found that RAPD markers were able to separate the accessions of $M$. sylvestris var. domestica used in their study based on their pedigree, but there were slight variations in the placement of some taxa between the dendrograms generated with the simple matching coefficient and Jaccard's coefficient of similarity. They attributed this to $\angle 50$ RAPD bands used in one of the analyses. However, the AMOVAR results in this study were very close for 24 RAPD markers and the entire dataset of 43 RAPD bands suggesting that $\approx 24$ RAPD bands are enough for partitioning the variation within the germplasm but more than 43 RAPD markers may be needed for determining relationships among the genotypes.

In conclusion, RAPD markers are a very useful tool for assessing genetic diversity in apples. It was relatively simple to generate a large number of polymorphisms to determine genetic relationships. The use of automation to run routine reactions enhances the reliability of the technique. However, due to the outbreeding nature of apples and the dominant nature of inheritance of RAPD markers, it may be useful to explore other fully informative molecular markers such as RFLPs or SSRs for a finer resolution of the relationships between the apple genotypes. Also, it would be useful to explore other methods for estimating similarities or distances than the shared band method.

We have demonstrated that old varieties are potential sources of diversity in apples, in addition to the wild species and current cultivars. The practice of grouping families into sublines is very popular in forestry. It is aimed at controlling inbreeding by restricting crosses within sublines in each generation. The level of variation obtained in this study suggests that, inbreeding is probably not a problem in apples and sublining the germplasm may not be necessary. If these results are confirmed by fully informative markers we may be able to merge the sublines into one population in the next generation to reduce costs and simultaneously maximize our selection and breeding efficiency.

\section{Literature Cited}

Becker, R.A., J.M. Chambers, and A.R.Wilks. 1988. The new S language, a programming environment for data analysis and graphics. Wadsworth and Brooks/Cole Advance Books and Software, Pacific Grove, Calif.

Bretting, P.K. and W.P. Widrlechner. 1995. Genetic markers and plant genetic resource management. Plant Breeding Rev. 13:11-86.

Caetano-Anolles,G., B.J. Bassam, and P.M. Gresshof. 1991. DNA amplification fingerprinting using very short arbitrary oligonucleatide primers. Biotechnology 9:553-557.

Chambers, J. and T. Hastie. 1992. Statistical models in S. Wadsworth and Brooks/Cole Advance Books and Software, Pacific Grove, Calif. 
Dunemann, F., R. Kahnau, and H. Schmidt. 1994. Genetic relationships in Malus evaluated by RAPD 'fingerprinting' of cultivars and wild species. Plant Breeding 113:150-159.

Excoffier, L., P.E. Smouse, and J.M. Quattro. 1992. Analysis of molecular variance inferred from metric distances among DNA haplotypes: Application to human mitochondrial DNA restriction data. Genet. Soc. Amer. 131:479-491.

Gardiner, S.E., H.C.M. Bassett, C. Madie, and D.A.M. Noiton. 1996b. Isozyme, randomly amplified polymorphic DNA (RAPD), and restriction fragment length polymorphism (RFLP) markers used to deduce a putative parent for the 'Braeburn' apple. J. Amer. Soc. Hort. Sci 121:996-1001.

Gardiner, S.E., H.C.M. Bassett, D.A.M. Noiton, V.G. Bus, M.E. Hofstee, A.G. White, R.D. Ball, R.L.S. Forster, and E.H.A. Rikkerink. 1996a. A detailed linkage map around an apple scab resistance gene demonstrates that two disease resistance classes both carry the $\mathrm{V} f$ gene. Theor. Appl. Genet. 93:485-493.

Gardiner, S.E., E.H.A. Rikkerink, and V.G.M. Bus. 1999. Use of DNA markers for introgression of resistance genes derived from wild relatives and for fingerprinting apple varieties. Proc. First Commonwealth Sci. Forum, Goa, India, 23-26 Sept. 1999.

Hamrick, JL. 1994. Genetic diversity and conservation in tropical forests, p. 1-9. In: R.M. Drysdale, S.E.T. John, and A.C. Yapa (eds.). Proc. Intl. Symp. on Genetic Conservation and Production of Tropical Forest Tree Seed. ASEAN-Canada Forest Tree Seed Centre Project, Muak-Lek, Saraburi, Thailand.

Harada, T., K. Matsukawa, T. Sato, R. Ishikawa, U. Niizeki, and K. Saito. 1993. DNA-RAPDs detect genetic variation and paternity in Malus. Euphytica 65:87-91.

Hedrick, P. 1992. Shooting the RAPDs. Nature (London) 355:679-680. Hokanson, S.C., J.R. McFerson, P.L. Forsline, W.F. Lamboy, J.J. Luby, A.D. Djangaliev, and H.S. Aldwinckle. 1997. Collecting and managing wild Malus germplasm in its centre of diversity. HortScience 32:173-176.

Hokanson, S.C., A.K. Szewc-McFadden, W.F. Lamboy, and J.R. McFerson. 1998. Microsatellite (SSR) markers reveal genetic identities, genetic diversity and relationships in a Malus $\times$ domestica Borkh. core subset collection. Theor. Appl. Genet. 97:671-683.

$\mathrm{Hu}, \mathrm{J}$ and C.F. Quiros. 1991. Identification of broccoli and cauliflower cultivars with RAPD markers. Plant Cell Rpt. 10:505-511.

Juniper, B.E., R.Watkins, and S.A. Harris. 1999. The origin of apple. Acta Hort. 484:27-33.

Kazan, K., J.M. Manners, and D.F. Cameron. 1993. Genetic variation in agronomically important species of Stylosanthes determined using random amplified polymorphic DNA markers. Theor. Appl. Genet. 85:901-904.

Koller, B., A. Lehmann, J.M. Mcdermott, and C. Gessler. 1993. Identi- fication of apple cultivars using RAPD markers. Theor. Appl. Genet. 85:901-904.

Korban, S.S. and R.M. Skirvin. 1984. Nomenclature of the cultivated apple. HortScience 19:177-180.

Kresovich, S., J.G.K. Williams, J.R. McFerson, E.J. Routman, and B.A. Schaal. 1992. Characterisation of genetic identities and relationships of Brassica oleraceae L. via random amplified polymorphic DNA assay. Theor. Appl. Genet. 85:190-196.

Lamboy, W.F., J. Yu, P.L. Forsline, and N.F. Weeden. 1996. Partitioning of Allozyme diversity in wild populations of Malus sieversii L. and implications for germplasm collection. J. Amer. Soc. Hort. Sci. 121:982987.

Lee, M. 1995. DNA markers and plant breeding programs. Adv. Agron. 55:265-344.

Noiton, D. and P. Alspach. 1996. Founding clones, inbreeding, coancestry, and status number of modern apple cultivars. J. Amer. Soc. Hort. Sci. 121:773-782.

Noiton, D., M. Hofstee, P. Alspach, L. Brewer, and C. Howard. 1999. Increasing genetic diversity for apple breeding: A preliminary report. Acta Hort. 484:105-107.

Noiton, D. and C.J.A Shelbourne. 1992. Quantitative genetics in an apple breeding strategy. Euphytica 60:213-219.

Provan, J., W. Powell, and R. Waugh. 1996. Microsatellite analysis of relationships within cultivated potato (Solanum tuberosum $\mathrm{L}$ ). Theor. Appl. Genet. 92:1078-1084.

Riesberg, L. 1996. Homology among RAPD fragments in interspecific comparisons. Mol. Ecol. 5:99-105.

Smith, M.W.G. 1971. National apple register of the United Kingdom. Ministry of Agriculture, Fisheries and Food, London.

Statistical Sciences, Inc., 1995. S-PLUS user's manual, version 3.4 for unix. Stat. Sci., Inc., Seattle, Wash.

Way, R.D., H.S. Aldwinckle, R.C. Lamb, A. Rejman, S. Sansavini, T. Shen, R. Watkins, M.N. Westwood, and Y. Yoshida. 1990. Apples (Malus), p. 5-62. In: J.N. Moore and J.R. Ballington (eds.). Genetic resources of temperate fruit and nut crops. vol. 1. Intl. Soc. Hort. Sci., Wageningen, The Netherlands.

Welsh, J. and M. McClelland. 1990. Fingerprinting genomes using PCR with arbitrary primers. Nucleic Acids Res. 18:7213-7218.

Welsh, J. and M. McClelland. 1991. Genomic fingerprinting using arbitrary primed PCR and a matrix of pairwise combinations of primers. Nucleic Acids Res. 19:5275-5279.

Williams, J.G.K., A.R. Kubelik, K.J. Livak, J.A. Rafalski, and S.V. Tingey. 1990. DNA polymorphisms amplified by arbitrary primers are useful as genetic markers. Nucleic Acids Res. 18:6531-6535.

Zhou, Z.Q. and Y.N. Li. 2000. The RAPD evidence for the phylogenetic relationship of the closely related species of cultivated apple. Genet. Res. Crop Evolution 47:353-357. 